\title{
Cellular senescence in cancer treatment: friend or foe?
}

\author{
Pascal Kahlem, ${ }^{1}$ Bernd Dörken, ${ }^{1,2}$ and Clemens A. Schmitt ${ }^{1,2}$ \\ ${ }^{1}$ Humboldt University, Charité, Department of Hematology, Oncology, and Tumor Immunology, Berlin, Germany \\ ${ }^{2}$ Max Delbrück Center for Molecular Medicine, Berlin, Germany
}

\begin{abstract}
Damage to DNA, the prime target of anticancer therapy, triggers programmed cellular responses. In addition to apoptosis, therapy-mediated premature senescence has been identified as another drug-responsive program that impacts the outcome of cancer therapy. Here, we discuss whether induction of senescence is a beneficial or, rather, a detrimental consequence of the therapeutic intervention.
\end{abstract}

J. Clin. Invest. 113:169-174 (2004). doi:10.1172/JCI200420784.

Achieving lasting remissions in patients suffering from nonlocalized malignancies remains the central problem of clinical oncology. Although the decades-old arsenal of classic anticancer treatment modalities such as surgery, chemotherapy, irradiation, and hormone ablation has been augmented by strategies including immunotherapy, gene therapy, inhibition of angiogenesis, hyperthermia, and a number of novel lesion-based approaches such as the administration of the Bcr-Abl kinase inhibitor STI-571, the goal to eradicate all cancer cells in a metastasized condition is rarely within reach. Anticancer treatment strategies may be insufficient for many reasons: potentially efficient therapies might not always find their way to virtually inaccessible tumor sites. Moreover, the multitude of tumor entities are known to differ remarkably in their susceptibilities to conventional DNA-damaging anticancer agents - particularly solid tumors, which often are largely refractory to chemotherapy or rapidly re-emerge from a remission. In addition, primarily susceptible tumors select for genetic defects during the course of therapy, which may render them resistant over time. While dose escalation can overcome the problem of insufficient chemosensitivity in some entities, its clinical applicability is limited by the severe toxicity codelivered to the normal cell compartment.

Traditional cytotoxic treatment strategies are driven by the assumption that quantitative execution of cell death is required to eliminate the malignant cell population.

\footnotetext{
Address correspondence to: Clemens A. Schmitt, Max Delbrück Center for Molecular Medicine, and Humboldt University, Charité, Department of Hematology, Oncology, and Tumor Immunology, Augustenburger Platz 1, 13353 Berlin, Germany. Phone: 49-30-450-553-687; Fax: 49-30-450-553-986;

E-mail: clemens.schmitt@charite.de.

Conflict of interest: The authors have declared that no conflict of interest exists.

Nonstandard abbreviations used: senescence-associated $\beta$ -

galactosidase (SA- $\beta$-Gal); ataxia telangiectasia mutated kinase (ATM); cyclin-dependent kinase (CDK); retinoblastoma protein (pRB); small interfering RNA (siRNA); mouse embryonic fibroblast (MEF).
}

Interestingly, strategies to blunt properties of malignant growth by disabling proliferation without primarily targeting cancer viability have been less recognized, although forcing cells to exit the cell cycle by an irreversible arrest should terminate their contribution to disease progression just as effectively. In fact, recent evidence underscores the theory that premature senescence may act as an acute, drug-inducible arrest program that may contribute to the outcome of cancer therapy $(1,2)$. In this Perspective, we will review the role of drug-induced effector programs and discuss to what extent induction of cellular senescence may be a beneficial result of anticancer therapy.

\section{Anticancer therapy induces programmed cellular responses}

Chemotherapy remains the mainstay in the treatment of systemic or metastasized malignancies. Although these highly toxic agents interfere with a plethora of cellular functions and may damage a variety of cellular structures, their pivotal cellular target is genomic DNA. It is now a well-accepted concept that drug-mediated DNA damage is not invariably lethal per se but provokes genetically encoded cellular responses. Hence, unrelated chemotherapeutic anticancer agents - in spite of their different pharmacological features and their individual target molecules participating in DNA replication and integrity - initiate common downstream mechanisms. Upon sensing DNA damage, cellular transducers activate pathways that either temporarily halt the cell cycle to allow the DNA repair machinery to fix the damage, or execute lethal programs such as apoptosis or mitotic catastrophe to restrain the damaged cell from further expansion (see ref. 3 for review). Ultimate, i.e., irreversible responses to DNA damage do not always determine the fate of cancer cells by programmed forms of cell death but may blunt their uncontrolled proliferative capacity by provoking a terminal cell-cycle arrest termed premature senescence (4-6). 
Moreover, recent reports demonstrated that tumor cell senescence is detectable following DNA-damaging treatment in vivo and significantly increases overall survival of the host $(1,2)$. In turn, the fact that different anticancer agents share genetic effector cascades renders the genetically encoded programs of apoptosis and senescence highly susceptible to inactivating mutations as a potential cause of chemoresistance. Hence, thorough dissection and mutational analysis of the pathways leading to cell death or cellular senescence are expected to identify specific genetic lesions that may be utilized by novel targeting therapies.

\section{Drug-inducible senescence is a p53- and $\mathrm{p} 16^{\mathrm{INK} 4 \mathrm{a}}$-controlled program}

Premature senescence recapitulates cellular and molecular features of replicative senescence (7), which is a safeguard program that limits the growth potential, but not necessarily the viability, of a dividing cell as a consequence of the progressive shortening of its telomeres. Senescent cells, arrested in the G1 phase of the cell cycle, typically appear flattened and enlarged with increased cytoplasmic granularity. In addition to the characteristic morphology, senescent cells display enhanced activity of senescence-associated $\beta$-galactosidase (SA- $\beta$-Gal) when assessed at an acidic $\mathrm{pH}(8,9)$. While refractory to mitogenic stimuli, senescent cells remain viable and metabolically active and possess a typical transcriptional profile that distinguishes them from quiescent cells (10). At the protein level, numerous regulators of cell-cycle progression, checkpoint control, and cellular integrity such as $\mathrm{p} 53$ or $\mathrm{p} 16^{\mathrm{INK} 4 \mathrm{a}}$ have been found to be induced in response to various pro-senescent stimuli $(4,11)$. Although the molecular mechanisms underlying the senescent phenotype remain largely unknown, there is increasing evidence that formation of heterochromatin in the vicinity of promoters that control gene expression related to cellcycle progression might be implicated in the maintenance of an irreversible growth arrest (12).

Extrinsic factors such as anticancer agents, $\gamma$-irradiation, or UV light have been shown to induce premature senescence as a DNA damage-mediated cellular stress response (4-6). DNA lesions are sensed and transduced via protein complexes involved in DNA maintenance and repair, associated with members of the PI3K superfamily that includes the ataxia telangiectasia mutated kinase (ATM) (13), the ATM-related kinase (ATR), and DNA-protein kinase, among others (14-18). Besides a network of other downstream substrates, these kinases directly or indirectly phosphorylate the gatekeeper of cellular integrity (19), the p53 protein, at certain residues. Although a cascade of posttranslational modifications has been proposed to control p53 activity in response to DNA damage (20), and different DNA-damaging stimuli such as UV light or $\gamma$-irradiation can produce distinguishable signatures of posttranslational p53 modifications (21), the actual contribution of distinct phospho-residues to p53-mediated DNA-damage responses is still under debate $(22,23)$. p5 3 controls a plethora of effector functions (24), and the precise mechanisms by which specific downstream pathways are regulated in response to p53 activation have not been elucidated yet $(25,26)$. While it is now clear that p53 participates not only in apoptosis but also in the induction of DNA damage-mediated senescence $(1,5$, $6)$, the signals that convert p53 from an apoptosis executor to a senescence inducer in response to anticancer therapy still need to be identified. Moreover, the posttranslational p53 modifications found in cells that entered replicative senescence shared only partial overlap with p53 modifications typically induced by DNA damage (27).

Comparable to p53, which functions as a fail-safe mediator, the cyclin-dependent kinase (CDK) inhibitor p16 ${ }^{\mathrm{INK} 4 a}$ has been implicated in both response to DNA damage and control of stress-induced senescence $(1,4$, $28,29)$. Although the molecular mechanism used by p16 $16^{\mathrm{INK} 4 a}$ to control not only a temporary but a permanent G1 arrest is largely unclear (30), $\mathrm{p} 16^{\mathrm{INK} 4 \mathrm{a}}$ responds to DNA damage in a delayed manner and appears to be indispensable for the maintenance of cellular senescence $(1,2)$. For example, treatment of normal human foreskin and lung fibroblasts with DNA-damaging drugs such as bleomycin or actinomycin D induced an irreversible cell-cycle arrest with a senescence-like phenotype including a transient upregulation of $\mathrm{p} 53$ and p21 protein levels, followed by increased $\mathrm{p} 16^{\text {INK4a }}$ protein expression and detectable SA- $\beta$-Gal activity (4). Similarly, exposure of adenocarcinoma cells to topoisomerase inhibitors produced premature senescence that was initially accompanied by overexpression of p53 and p21, whereby the subsequent overexpression of $\mathrm{p} 16^{\mathrm{INK} 4 a}$ persisted after drug withdrawal, highlighting the role of $\mathrm{p} 16^{\mathrm{INK} 4 \mathrm{a}}$ in maintenance of the growth arrest (2). In an in vivo model of drug-senescent mouse lymphomas, repeated anticancer therapy eventually selected against senescence-controlling genes such as the $\mathrm{p} 16^{\mathrm{INK} 4 \mathrm{a}}$-encoding INK4a alleles or $p 53$ genes, thereby producing relapse tumors that resumed growth in an aggressive manner (1). A broader drug screen based on several tumor cell lines demonstrated inducibility of a senescence-like arrest in numerous p53-proficient cancer cell lines in response to doxorubicin and other DNA-damaging agents, but it also suggested that p53independent pathways leading to senescence might exist when some p53-mutated cell lines were exposed to escalated doses of doxorubicin (5). Interestingly, not only DNA-damaging agents, but also compounds that primarily target microtubules or the differentiating agent retinoic acid, were found to promote an SA- $\beta-$ Galpositive arrest phenotype. Moreover, a senescence-like growth arrest was also observed after $\gamma$-irradiation of normal human diploid cells $(6,31)$. p53 deficiency, previously reported to disable apoptosis in response to $\gamma$-irradiation $(32,33)$, also accounted for impaired $\gamma$-irradiation-induced senescence in a human carcinoma cell line wherein senescence could be restored upon 
introduction of wild-type p53 (6). Thus, in a variety of different test conditions, DNA damage-induced senescence was confirmed as a $\mathrm{p} 53-$ and $\mathrm{p} 16^{\mathrm{INK} 4 \mathrm{a}}$-co-controlled safeguard program.

\section{Drug-induced senescence: substitute player or powerful reliever?}

The possibility of alternative outcomes in response to drug-induced DNA damage - apoptosis, mitotic catastrophe, cellular senescence, or simply necrosis - raises the question of whether additional stimuli or specific contextual scenarios determine the ultimate cell fate. Xenotransplant and transgenic mouse models have been used to visualize premature senescence as a quantitative response to anticancer agents in vivo $(1,5)$. Importantly, lymphomas generated in the $E \mu-m y c$ transgenic mouse model were prone to massive apoptosis as a default response following therapy with the alkylating agent cyclophosphamide, but they uniformly displayed premature senescence when apoptosis was blocked by overexpression of the strictly antiapoptotic mediator Bcl2 (1). Although mice harboring senescent lymphomas ultimately succumbed to their disease, they lived much longer than those bearing lymphomas with a defect in both apoptosis and senescence as a consequence of p53 loss. Given the rapid induction of apoptosis and the rather delayed detectability of an SA- $\beta$-Gal-positive long-term arrest in response to anticancer therapy in vivo, the data suggested that the senescence machinery may act as a back-up program to substitute for or to reinforce an insufficient apoptotic response (34). The actual impact of a fictitious population of cells that chose to primarily enter senescence is difficult to assess, since apoptotically competent tumors typically regress to a remission with no apparent residual tumor mass left. Senescent cells have also been detected in archival tumor samples from breast cancer patients who underwent neoadjuvant chemotherapy prior to surgical removal of their tumors. In contrast to untreated tumor samples, these samples revealed significant positive staining for SA- $\beta$-Gal activity associated with high expression of p16 $6^{\text {INK4a }}(2)$. Hence, intrinsically rather chemosensitive entities such as hematological malignancies may use senescence as a secondary mechanism, while typically less susceptible solid tumors might rely on premature senescence as the chief drugresponse program available.

According to many naturally occurring mutations in apoptosis-related genes, disruption of apoptosis is, at least in conjunction with certain oncogenic scenarios, a pivotal step in tumor development. Given the complex overlap between apoptosis and senescence as cellular fail-safe systems on one hand and tumor-suppressor mechanisms and drug-effector programs on the other hand, the capability to execute senescence might be disabled in cancer cells for numerous reasons. In fact, mutations in genes that control cellular senescence may not only be selected for during therapy but might have been acquired already during tumor development (1). Oncogenes such as activated ras that are known to provoke premature senescence as the primary fail-safe mechanism may rely on defects in this program as a prerequisite to a fully transformed phenotype $(28,35)$, possibly inactivating senescence as a drug response as well. Nevertheless, tumors that preserve both an intact apoptotic and a functional senescence program may display a particularly robust drug response consisting of acutely inducible cell death in a first phase, corroborated by delayed apoptosis out of senescence at a later point (Figure 1). Although apoptosis is a fast-acting response mode, little is known about the possibility that apoptosis-competent cells might be sent into senescence following DNA-damaging therapy - and whether a senescent tumor could ever undergo apoptosis upon an additional proapoptotic signal (36). Finally, senescence could be recruited as an amplifier mechanism to lock temporarily arrested tumor cells - with their reduced susceptibility to checkpoint-licensed apoptosis - into irreversible cytostasis.

\section{No effect without side effects}

The fate of apoptotic cells is determined by their acute disruption of metabolic processes, rapid disintegration, and engulfment by attracted macrophages. In stark contrast, induction of cellular senescence as a formally irreversible growth arrest results in the preservation of a potentially malignant cell population locked into a nondividing state, yet possessing at least some metabolic activity (8). Although apoptotic cells provoke rather little inflammatory reaction, tumorinfiltrating immune cells reportedly can recognize altered autoantigens presented by apoptotic cancer cells (37). To what extent altered senescent tumor cells that previously managed to escape immunosurveillance can now challenge an antitumor immune response requires further investigation (Figure 1). Correlative evidence points toward a link between dermal autoimmunity in the elder population and an agedependent increase of SA- $\beta$-Gal-positive keratinocytes in human skin samples (8).

Although it is likely that senescent cells will ultimately be cleared by phagocytosis, no "eat-me" signals, as recently described for apoptotic cells, have been identified yet for the senescent state (38). While senescent neutrophils, like apoptotic granulocytes (39), might ultimately face phagocytosis through a yet unknown recognition mechanism (40), focal enrichment of lysosome-related $\beta$-galactosidase activity at autodigestive vacuoles indicated that aged human fibroblasts arrested in replicative senescence may eventually eliminate themselves by autophagy (41). As a side effect of anticancer therapy, DNA damage can also force susceptible normal cells to enter an acute SA- $\beta$-Gal-positive arrest in vivo (S. Lee and C.A. Schmitt, unpublished observations). Hence, it is conceivable that senescent cancer and normal bystander cells reside for some time next to 


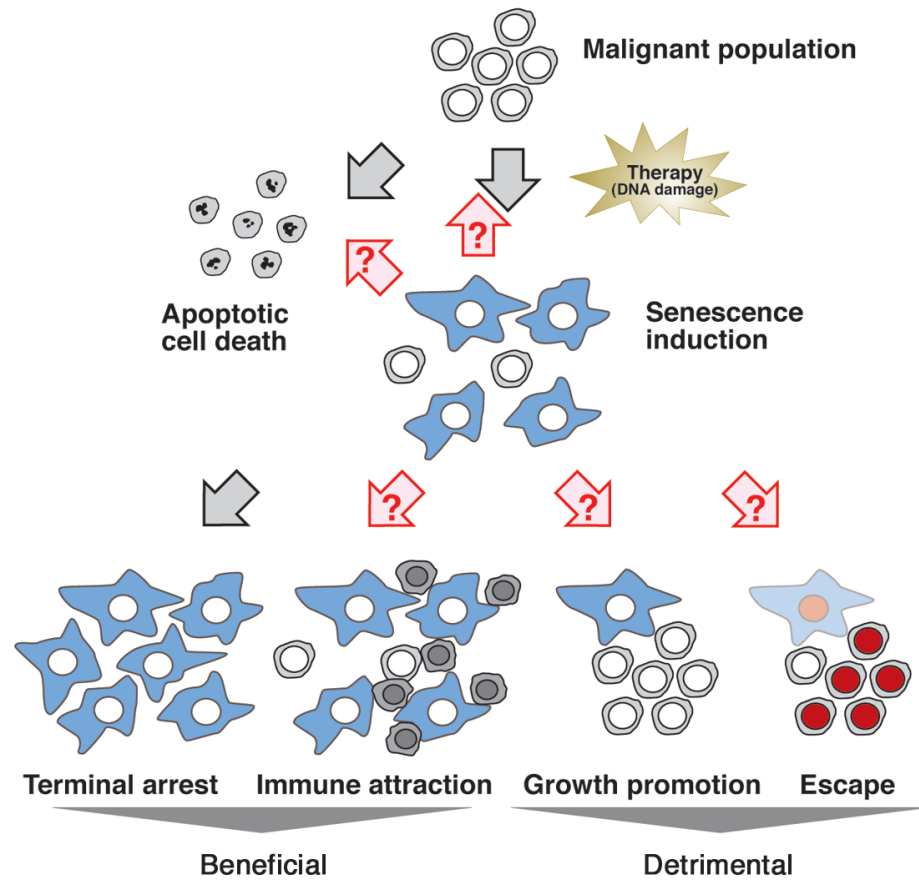

Figure 1

Drug-inducible senescence: friend or foe? In response to DNA-damaging agents, cancer cells can rapidly undergo apoptosis or may enter premature senescence as a potential back-up mechanism. Whether cells re-enter the cycle or execute apoptosis out of drug-mediated senescence remains unclear. A terminal arrest of the entire cancer cell population, possibly augmented through increased immunogenicity of senescent cells, is beneficial for the host. In contrast, feeder-like growth that reflects paracrine activity of senescent cells on their non-senescent neighbors, or escape from senescence based on acquired or preexisting mutations, is considered a detrimental outcome. their non-senescent malignant neighbors. In line with the role of irradiated fibroblasts as feeder cells, and with evidence reported by Waldman and colleagues, who described improved clonogenic growth in the presence of irradiation-arrested bystander cells (42), interspersed senescent - i.e., metabolically active - cells may support survival and growth of tumor cells in their vicinity. Campisi and coworkers demonstrated that senescent human fibroblasts stimulated proliferation of epithelial cell lines in vitro (43). Moreover, coimplantation of senescent fibroblasts together with preneoplastic epithelial cells in nude mice accelerated tumor formation in vivo, mainly via soluble factors secreted by the senescence-activated fibroblasts. Thus, the outcome of anticancer therapy is not only determined by a quantitative effect on cancer cells forced to irreversibly exit the cell cycle but may also depend on novel capabilities acquired by senescent cells that can impact their malignant neighbors in different ways (Figure 1). It is quite possible that, because of functionally compromised cell-cell interactions, senescent tumor cells can even exert a tumor-suppressive effect on their bystander cells.

\section{Rest in peace?}

Cellular senescence has become an attractive therapeutic concept because it qualitatively equals programmed cell death by excluding cells from active progression through the cell cycle. However, its therapeutic potential strongly relies on the irreversibility of this process. Unlike apoptosis, which acutely eliminates a potentially harmful cell, cellular senescence represents an operational change in a still-viable cell. Any cellular switch that could revert senescent cells into dividing cells implies the threat of a tumor relapse - at least as long as senescent cells have not been cleared by other processes such as phagocytosis. In fact, several experiments have provided evidence that senescence is a formally reversible process if proteins involved in its maintenance are lost. For example, reversal of replicative senescence in human lung fibroblasts was achieved via functional inactivation of both $\mathrm{p} 53$ and the retinoblastoma protein $(\mathrm{pRB})$ by the expression of simian virus 40 large $\mathrm{T}$ antigen protein, or, as an alternative, by a combination of p53 inactivation and knockdown of p16 ${ }^{\mathrm{INK} 4 a}$ expression using small interfering RNA (siRNA) molecules (44). In contrast, the mere suppression of $\mathrm{p} 53$ function proved already sufficient to revert the senescent phenotype of human foreskin fibroblasts (44). Likewise, siRNA-driven inactivation of p53 in mouse embryonic fibroblasts (MEFs) enabled the cells to resume proliferation out of replicative senescence (45). These findings are in accordance with the putative cooperativity of $\mathrm{p} 53$ and $\mathrm{p} 16^{\mathrm{INK} 4 \mathrm{a}}-$ and possibly $\mathrm{pRB}-$ in the induction and maintenance of premature senescence in vivo (1). Accordingly, the acute inactivation of $\mathrm{pRB}$ via recombinase-mediated gene deletion in MEFs that entered senescence upon replicative exhaustion or in response to oncogenic ras allowed the cells to reemerge from the arrested phenotype (46). However, it needs to be shown that this cell-cycle re-entry truly reflects restored proliferative capacity and will not simply promote cell death by apoptosis or mitotic catastrophe within a few additional divisions.

Importantly, these experimental scenarios have not formally tested whether drug-inducible senescence is a reversible process as well. In the p53-and pRB-deficient cell line Saos-2, premature senescence - in addition to substantial cell death - was observed in response to doxorubicin treatment, suggesting a p53- 
and pRB-independent pathway to senescence (5). Furthermore, overexpression of a temperature-sensitive pRB transgene in Saos- 2 cells produced a senescent phenotype. As a result of transgene inactivation, reinitiation of DNA synthesis was observed, and the cells underwent apoptotic cell death via the p53 homologue $\mathrm{p} 73$, indicating that active $\mathrm{pRB}$ is required to maintain the senescent state of these cells (47). This somewhat artificial setting underscores the complex wiring of signaling pathways into senescence, their relative responsiveness to different stimuli, their dependency on the cell type, and their sensitivity to experimental approaches based on nonphysiological overexpression of candidate regulators. Nevertheless, some of the experiments demonstrating successful reversal of cellular senescence that senescent cancer cells could actually encounter in vivo. While the acquisition of spontaneous mutations that disable $\mathrm{p} 53$ or pRB in a resting cell without DNA replication seems rather unlikely, epigenetic changes, for example, promoter methylation to silence $\mathrm{p} 16^{\mathrm{INK} 4 \mathrm{a}}$ expression, might occur in senescent cells (Figure 1).

\section{Exploiting cellular senescence for cancer therapy}

The uncertainties regarding control and irreversibility of drug-induced senescence raise concerns as to what extent this effector mechanism reflects a desirable outcome of cancer therapy, particularly in light of therapyinducible apoptosis as the alternate and possibly safer outcome in response to DNA damage. However, an intact apoptotic machinery is often unavailable in established malignancies. Since anticancer agents kill mainly by apoptotic cell death and, in turn, achieve little clinical efficacy in the presence of apoptotic defects (see, for example, refs. 48-52), promising treatment alternatives must use effector mechanisms that do not rely on an intact apoptotic machinery. Importantly, in vivo analyses of treatment responses in primary lymphomas harboring defined genetic lesions demonstrated that induction of senescence despite the presence of an apoptotic block improved the outcome of anticancer therapy (1) regardless of a potential reversal or possible emergence of preexisting escape mutants at a later time.

Appropriate test systems are critical to elucidate the complex implications of drug-inducible senescence. Not surprisingly, drug sensitivity assays performed on primary tumor material in culture unveiled that adaptation to the nonphysiological culture conditions selected for apoptotic defects and chemoresistance (50). Paradoxically, many cell lines retain the ability to enter senescence following drug exposure in vitro (5), although inactivation of the terminal-arrest program appears to be a key prerequisite for any primary tumor during successful establishment as a continuous cell line. Irrespective of the technical inducibility of a senescence-like phenotype in culture-adapted cells, a Petri dish setting cannot mimic the complexity and interactivity of a natural tumor environment in vivo. Hence, many of the questions raised about the role of drug-induced senescence need to be addressed in vivo using physiological model systems. Tractable mouse models of cancer, such as the transgenic E $\mu$-myc lymphoma model (1), in alliance with sophisticated genetic tools will allow researchers to dissect the pathways and the impact of drug-inducible senescence in vivo. Moreover, large-scale analyses of the transcriptome and proteome of primary human tumor samples will expand our understanding of the molecular mechanisms that underlie drug responses in sensitive and resistant conditions.

Given the impact of apoptotic defects on tumor biology and treatment outcome, it is a research priority to invent small compound- or gene therapy-based approaches that may resensitize cancer cells to death signals. Likewise, one can envision lesion-based strategies to restore a defective senescence response. Ultimately, direct activation of pro-senescent pathways without induction of deleterious DNA damage seems to be a particularly appealing concept. For instance, cDNA microarray analysis of human diploid fibroblasts revealed that cGMP synthesis was inhibited during replicative senescence. Exposure of tumor cells to a guanylate cyclase inhibitor induced, by activation of the CDK inhibitor p21, a senescent phenotype that was independent of its upstream regulator p53, indicating that induction of $\mathrm{p} 21$ activity might be sufficient to halt cell proliferation, even in the absence of functional p53 and without an additional DNA-damage signal (53). Furthermore, a synthetic inhibitor of CDK 4 - possibly mimicking the role of $\mathrm{p} 16^{\mathrm{INK} 4 a}$ to maintain a senescent phenotype - also produced a DNA damage-independent form of premature senescence in cells lacking proper $\mathrm{p} 16^{\mathrm{INK} 4 \mathrm{a}}$ expression and inhibited the growth of xenotransplant tumors in mice (54). Inactivation of the papilloma virus oncoproteins $\mathrm{E} 6$ and $\mathrm{E} 7$, which deregulate the $\mathrm{p} 53$ and $\mathrm{Rb}$ proteins, respectively, by siRNA molecules restored cellular senescence in cervical cancer cells (55).

Cellular senescence and its potential use as a drugeffector program remains a complex biological phenomenon with unknown significance in cancer therapy. Whether cellular senescence is rather friend or foe most likely depends on accompanying lesions, first of all in apoptotic response programs, and on the cellular context. In further preclinical investigations, it will be of particular interest to explore therapies that do not deliver devastating DNA damage to the cell, that do not rely on functional DNA-damage transducer systems, and that do not target pathways already mutated to cancel apoptosis, but that directly prompt a senescence response.

\section{Acknowledgments}

Research in the authors' laboratory is supported in part by grants from the Deutsche Forschungsgemeinschaft and Deutsche Krebshilfe. We apologize to our colleagues whose work has not been covered in this article because of space limitations. We thank Soyoung Lee for helpful discussions and editorial advice. 
1. Schmitt, C.A., et al. 2002. A senescence program controlled by p53 and p16INK4a contributes to the outcome of cancer therapy. Cell. 109:335-346.

2. te Poele, R.H., Okorokov, A.L., Jardine, L., Cummings, J., and Joel, S.P. 2002. DNA damage is able to induce senescence in tumor cells in vitro and in vivo. Cancer Res. 62:1876-1883.

3. Roninson, I.B., Broude, E.V., and Chang, B.D. 2001. If not apoptosis, then what? Treatment-induced senescence and mitotic catastrophe in tumor cells. Drug Resist. Updat. 4:303-313.

4. Robles, S.J., and Adami, G.R. 1998. Agents that cause DNA double strand breaks lead to p16INK4a enrichment and the premature senescence of normal fibroblasts. Oncogene. 16:1113-1123.

5. Chang, B.D., et al. 1999. A senescence-like phenotype distinguishes tumor cells that undergo terminal proliferation arrest after exposure to anticancer agents. Cancer Res. 59:3761-3767.

6. Suzuki, K., et al. 2001. Radiation-induced senescence-like growth arrest requires TP53 function but not telomere shortening. Radiat. Res. 155:248-253.

7. Hayflick, L., and Moorhead, P.S. 1961. The serial cultivation of human diploid cell strains. Exp. Cell Res. 25:585-621.

8. Dimri, G.P., et al. 1995. A biomarker that identifies senescent human cells in culture and in aging skin in vivo. Proc. Natl. Acad. Sci. U. S. A. 92:9363-9367.

9. Campisi, J., Dimri, G., and Hara, E. 1996. Control of replicative senescence. In Handbook of the biology of aging. S.A.J. Rowe, editor. Academic Press. New York, New York, USA. 121-149.

10. Shelton, D.N., Chang, E., Whittier, P.S., Choi, D., and Funk, W.D. 1999. Microarray analysis of replicative senescence. Curr. Biol. 9:939-945.

11. Serrano, M. 1997. The tumor suppressor protein p16INK4a. Exp. Cell Res. 237:7-13.

12. Narita, M., et al. 2003. Rb-mediated heterochromatin formation and silencing of E2F target genes during cellular senescence. Cell. 113:703-716.

13. Canman, C.E., and Lim, D.S. 1998. The role of ATM in DNA damage responses and cancer. Oncogene. 17:3301-3308.

14. Tee, A.R., and Proud, C.G. 2000. DNA-damaging agents cause inactivation of translational regulators linked to mTOR signalling. Oncogene. 19:3021-3031

15. Abraham, R.T. 2001. Cell cycle checkpoint signaling through the ATM and ATR kinases. Genes Dev. 15:2177-2196.

16. Durocher, D., and Jackson, S.P. 2001. DNA-PK, ATM and ATR as sensors of DNA damage: variations on a theme? Curr. Opin. Cell Biol. 13:225-231.

17. Rouse, J., and Jackson, S.P. 2002. Interfaces between the detection, signaling, and repair of DNA damage. Science. 297:547-551.

18. Dent, P., Yacoub, A., Fisher, P.B., Hagan, M.P., and Grant, S. 2003. MAPK pathways in radiation responses. Oncogene. 22:5885-5896.

19. Levine, A.J. 1997. p53, the cellular gatekeeper for growth and division. Cell. 88:323-331.

20. Sakaguchi, K., et al. 1998. DNA damage activates p53 through a phosphorylation-acetylation cascade. Genes Dev. 12:2831-2841.

21. Chehab, N.H., Malikzay, A., Stavridi, E.S., and Halazonetis, T.D. 1999. Phosphorylation of Ser-20 mediates stabilization of human p53 in response to DNA damage. Proc. Natl. Acad. Sci. U. S. A. 96:13777-13782.

22. Shieh, S.Y., Taya, Y., and Prives, C. 1999. DNA damage-inducible phosphorylation of p53 at N-terminal sites including a novel site, Ser20, requires tetramerization. EMBO J. 18:1815-1823.

23. Chao, C., Saito, S., Anderson, C.W., Appella, E., and Xu, Y. 2000. Phosphorylation of murine $\mathrm{p} 53$ at ser- 18 regulates the $\mathrm{p} 53$ responses to DNA damage. Proc. Natl. Acad. Sci. U. S. A. 97:11936-11941.

24. Vousden, K.H., and Lu, X. 2002. Live or let die: the cell's response to p53. Nat. Rev. Cancer. 2:594-604.

25. Oda, K., et al. 2000. p53AIP1, a potential mediator of p53-dependent apoptosis, and its regulation by Ser-46-phosphorylated p53. Cell. 102:849-862.

26. D'Orazi, G., et al. 2002. Homeodomain-interacting protein kinase-2 phosphorylates p53 at Ser 46 and mediates apoptosis. Nat. Cell Biol. 4:11-19.

27. Webley, K., et al. 2000. Posttranslational modifications of p53 in replicative senescence overlapping but distinct from those induced by DNA damage. Mol. Cell. Biol. 20:2803-2808.

28. Serrano, M., Lin, A.W., McCurrach, M.E., Beach, D., and Lowe, S.W. 1997. Oncogenic ras provokes premature cell senescence associated with accumulation of p53 and p16INK4a. Cell. 88:593-602.

29. Shapiro, G.I., Edwards, C.D., Ewen, M.E., and Rollins, B.J. 1998. p16INK4A participates in a G1 arrest checkpoint in response to DNA damage. Mol. Cell. Biol. 18:378-387.

30. Agami, R., and Bernards, R. 2000. Distinct initiation and maintenance mechanisms cooperate to induce G1 cell cycle arrest in response to DNA damage. Cell. 102:55-66.

31. Seidita, G., Polizzi, D., Costanzo, G., Costa, S., and Di Leonardo, A. 2000. Differential gene expression in p53-mediated $\mathrm{G}(1)$ arrest of human fibroblasts after gamma-irradiation or N-phosphoacetyl-L-aspartate treatment. Carcinogenesis. 21:2203-2210.

32. Lowe, S.W., Schmitt, E.M., Smith, S.W., Osborne, B.A., and Jacks, T. 1993 p53 is required for radiation-induced apoptosis in mouse thymocytes. Nature. 362:847-849.

33. Schmitt, C.A., Wallace-Brodeur, R.R., Rosenthal, C.T., McCurrach, M.E., and Lowe, S.W. 2000. DNA damage responses and chemosensitivity in the E mu-myc mouse lymphoma model. Cold Spring Harb. Symp. Quant. Biol. 65:499-510.

34. Lee, S., and Schmitt, C.A. 2003. Chemotherapy response and resistance. Curr. Opin. Genet. Dev. 13:90-96.

35. Brookes, S., et al. 2002. INK4a-deficient human diploid fibroblasts are resistant to RAS-induced senescence. EMBO J. 21:2936-2945.

36. Wang, E. 1995. Senescent human fibroblasts resist programmed cell death, and failure to suppress bcl2 is involved. Cancer Res. 55:2284-2292.

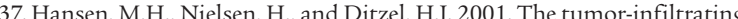
$\mathrm{B}$ cell response in medullary breast cancer is oligoclonal and directed against the autoantigen actin exposed on the surface of apoptotic cancer cells. Proc. Natl. Acad. Sci. U. S. A. 98:12659-12664.

38. Lauber, K., et al. 2003. Apoptotic cells induce migration of phagocytes via caspase-3-mediated release of a lipid attraction signal. Cell. 113:717-730.

39. Savill, J.S., et al. 1989. Macrophage phagocytosis of aging neutrophils in inflammation. Programmed cell death in the neutrophil leads to its recognition by macrophages. J. Clin. Invest. 83:865-875.

40. Murphy, J.F., McGregor, J.L., and Leung, L.L. 1998. Senescent human neutrophil binding to thrombospondin (TSP): evidence for a TSP-independent pathway of phagocytosis by macrophages. Br. J. Haematol. 102:957-964

41. Gerland, L.M., et al. 2003. Association of increased autophagic inclusions labeled for beta-galactosidase with fibroblastic aging. Exp. Geron tol. 38:887-895.

42. Waldman, T., et al. 1997. Cell-cycle arrest versus cell death in cancer therapy. Nat.Med. 3:1034-1036.

43. Krtolica, A., Parrinello, S., Lockett, S., Desprez, P.Y., and Campisi, J. 2001. Senescent fibroblasts promote epithelial cell growth and tumorigenesis: a link between cancer and aging. Proc. Natl. Acad. Sci. U. S. A. 98:12072-12077.

44. Beausejour, C.M., et al. 2003. Reversal of human cellular senescence: roles of the p53 and p16 pathways. EMBO J. 22:4212-4222.

45. Dirac, A.M., and Bernards, R. 2003. Reversal of senescence in mouse fibroblasts through lentiviral suppression of p53. J. Biol. Chem. 278:11731-11734.

46. Sage, J., Miller, A.L., Perez-Mancera, P.A., Wysocki, J.M., and Jacks, T. 2003. Acute mutation of retinoblastoma gene function is sufficient for cell cycle re-entry. Nature. 424:223-228.

47. Alexander, K., Yang, H.S., and Hinds, P.W. 2003. pRb inactivation in senescent cells leads to an E2F-dependent apoptosis requiring p73. Mol. Cancer Res. 1:716-728.

48. Schmitt, C.A., McCurrach, M.E., de Stanchina, E., Wallace-Brodeur, R.R., and Lowe, S.W. 1999. INK4a/ARF mutations accelerate lymphomagenesis and promote chemoresistance by disabling p53. Genes Dev. 13:2670-2677.

49. Dohner, H., et al. 2000. Genomic aberrations and survival in chronic lymphocytic leukemia. N. Engl. J. Med. 343:1910-1916.

50. Schmitt, C.A., Rosenthal, C.T., and Lowe, S.W. 2000. Genetic analysis of chemoresistance in primary murine lymphomas. Nat. Med. 6:1029-1035.

51. Chang, J.C., et al. 2003. Gene expression profiling for the prediction of therapeutic response to docetaxel in patients with breast cancer. Lancet. 362:362-369.

52. Holleman, A., Den Boer, M.L., Kazemier, K.M., Janka-Schaub, G.E., and Pieters, R. 2003. Resistance to different classes of drugs is associated with impaired apoptosis in childhood acute lymphoblastic leukaemia. Blood. 102:4541-4546.

53. Lodygin, D., Menssen, A., and Hermeking, H. 2002. Induction of the Cdk inhibitor p21 by LY83583 inhibits tumor cell proliferation in a p53-independent manner. J. Clin. Invest. 110:1717-1727. doi:10.1172/ JCI200216588.

54. Soni, R., et al. 2001. Selective in vivo and in vitro effects of a small molecule inhibitor of cyclin-dependent kinase 4. J. Natl. Cancer Inst. 93:436-446.

55. Hall, A.H., and Alexander, K.A. 2003. RNA interference of human papillomavirus type 18 E6 and E7 induces senescence in HeLa cells. J. Virol. 77:6066-6069. 